\title{
Oscillation of fourth-order strongly noncanonical differential equations with delay argument
}

\author{
B. Baculikova ${ }^{1 *}$ and J. Dzurina ${ }^{1}$
}

"Correspondence:

blanka.baculikova@tuke.sk

${ }^{1}$ Department of Mathematics,

Faculty of Electrical Engineering and Informatics, Technical University of

Košice, Košice, Slovakia

\section{Abstract}

The aim of this paper is to study oscillatory properties of the fourth-order strongly noncanonical equation of the form

$$
\left(r_{3}(t)\left(r_{2}(t)\left(r_{1}(t) y^{\prime}(t)\right)^{\prime}\right)^{\prime}\right)^{\prime}+p(t) y(\tau(t))=0,
$$

where $\int^{\infty} \frac{1}{r_{i}(s)} \mathrm{d} s<\infty, i=1,2,3$. Reducing possible classes of the nonoscillatory solutions, new oscillatory criteria are established.

MSC: 34K11;34C10

Keywords: Noncanonical operator; Fourth order differential equations; Oscillation

\section{Introduction}

In the paper, we consider the fourth-order delay differential equation

$$
\left(r_{3}(t)\left(r_{2}(t)\left(r_{1}(t) y^{\prime}(t)\right)^{\prime}\right)^{\prime}\right)^{\prime}+p(t) y(\tau(t))=0,
$$

where $r_{i} \in C^{(4-i)}\left(t_{0}, \infty\right), r_{i}(t)>0, i=1,2,3, p(t) \in C\left(t_{0}, \infty\right), p(t)>0, \tau(t) \in C\left(t_{0}, \infty\right), \tau(t) \leq$ $t, \tau^{\prime}(t)>0$, and $\tau(t) \rightarrow \infty$ as $t \rightarrow \infty$.

By a solution of Eq. $(E)$ we mean all continuous functions $y(t)$ for which

$$
\left(r_{3}(t)\left(r_{2}(t)\left(r_{1}(t) y^{\prime}(t)\right)^{\prime}\right)^{\prime}\right)^{\prime} \in C\left(\left[T_{y}, \infty\right)\right), \quad T_{y} \geq t_{0},
$$

exist and satisfy Eq. $(E)$ on $\left[T_{y}, \infty\right)$. We consider only those solutions $y(t)$ of $(E)$ which satisfy $\sup \{|y(t)|: t \geq T\}>0$ for all $T \geq T_{y}$. We assume that $(E)$ possesses such a solution. A solution of $(E)$ is called oscillatory if it has arbitrarily large zeros on $\left[T_{y}, \infty\right)$ and otherwise it is called nonoscillatory. Equation $(E)$ is said to be oscillatory if all its solutions are oscillatory.

Throughout the paper it is supposed that Eq. $(E)$ is strongly noncanonical, that is,

$$
\int^{\infty} \frac{1}{r_{i}(s)} \mathrm{d} s<\infty, \quad i=1,2,3
$$

(c) The Author(s) 2019. This article is distributed under the terms of the Creative Commons Attribution 4.0 International License (http://creativecommons.org/licenses/by/4.0/), which permits unrestricted use, distribution, and reproduction in any medium, provided you give appropriate credit to the original author(s) and the source, provide a link to the Creative Commons license, and indicate if changes were made. 
Fourth-order differential equations naturally appear in models concerning physical, biological, and chemical phenomena, such as, for instance, problems of elasticity, deformation of structures, or soil settlement, see, for example, [2]. In mechanical and engineering problems, questions concerning the existence of oscillatory solutions play an important role. During the past decades, there has been a constant interest in obtaining sufficient conditions for oscillatory properties of different classes of fourth-order differential equations with deviating argument, see [2, 3, 6, 8-20].

In general, there are two approaches for the investigation of higher-order differential equations with noncanonical operators. One method requires to find a canonical representation of studied equation with closed form formulas for coefficients. For details, see $[1,4,5,7]$. The second approach is to establish the conditions that reduce the number of possible classes of nonoscillatory solutions and consequently to find conditions for oscillation of $(E)$. Our method belongs to the second one and yields easily verifiable oscillation criteria.

\section{Preliminary results}

Throughout the paper we assume that (1.1) holds, and so we can employ the notation

$$
\pi_{i}(t)=\int_{t}^{\infty} \frac{1}{r_{i}(s)} \mathrm{d} s, \quad \pi_{i j}(t)=\int_{t}^{\infty} \frac{1}{r_{i}(s)} \pi_{j}(s) \mathrm{d} s
$$

and

$$
\pi_{i j k}(t)=\int_{t}^{\infty} \frac{1}{r_{i}(s)} \pi_{j k}(s) \mathrm{d} s,
$$

where $i, j, k \in\{1,2,3\}$ are mutually different. To simplify the writing of quasi-derivatives, we denote

$$
L_{1} y(t)=r_{1}(t) y^{\prime}(t), \quad L_{i+1} y(t)=r_{i+1}(t) L_{i}^{\prime} y(t), \quad i=1,2,3,
$$

where formally $r_{4}(t) \equiv 1$. We start with the following auxiliary results which are elementary but very useful.

Lemma 1 Let (1.1) hold. Then

$$
\pi_{i j}(t)+\pi_{j i}(t)=\pi_{i}(t) \pi_{j}(t)
$$

Proof Since

$$
\left(\pi_{i}(t) \pi_{j}(t)\right)^{\prime}=-\frac{\pi_{j}(t)}{r_{i}(t)}-\frac{\pi_{i}(t)}{r_{j}(t)}
$$

an integration of this equality from $t$ to $\infty$ yields

$$
\pi_{i}(t) \pi_{j}(t)=\int_{t}^{\infty} \frac{1}{r_{i}(s)} \pi_{j}(s) \mathrm{d} s+\int_{t}^{\infty} \frac{1}{r_{j}(s)} \pi_{i}(s) \mathrm{d} s=\pi_{i j}(t)+\pi_{j i}(t) .
$$


Lemma 2 Let (1.1) hold. Then

$$
\pi_{123}(t)+\pi_{32}(t) \pi_{1}(t)-\pi_{3}(t) \pi_{12}(t)=\pi_{321}(t) .
$$

Proof Proof of this lemma is similar to that of Lemma 1 and so it can be omitted.

It follows from a generalization of lemma of Kiguradze [9] that the set of positive solutions of $(E)$ has the following structure.

Lemma 3 Assume that $y(t)$ is a positive solution of $(E)$. Then $y(t)$ satisfies one of the following conditions:

$\left(N_{1}\right): L_{1} y(t)>0, L_{2} y(t)>0, L_{3} y(t)>0, L_{4} y(t)<0$,

$\left(N_{2}\right): L_{1} y(t)>0, L_{2} y(t)>0, L_{3} y(t)<0, L_{4} y(t)<0$,

$\left(N_{3}\right): L_{1} y(t)>0, L_{2} y(t)<0, L_{3} y(t)<0, L_{4} y(t)<0$,

$\left(N_{4}\right): L_{1} y(t)>0, L_{2} y(t)<0, L_{3} y(t)>0, L_{4} y(t)<0$,

$\left(N_{5}\right): L_{1} y(t)<0, L_{2} y(t)>0, L_{3} y(t)>0, L_{4} y(t)<0$,

$\left(N_{6}\right): L_{1} y(t)<0, L_{2} y(t)<0, L_{3} y(t)>0, L_{4} y(t)<0$,

$\left(N_{A}\right): L_{1} y(t)<0, L_{2} y(t)>0, L_{3} y(t)<0, L_{4} y(t)<0$,

$\left(N_{B}\right): L_{1} y(t)<0, L_{2} y(t)<0, L_{3} y(t)<0, L_{4} y(t)<0$.

The first two results are intended to reduce the number of classes that will be investigated.

\section{Theorem 1 If}

$$
\int_{t_{1}}^{\infty} \pi_{32}(s) p(s) \mathrm{d} s=\infty
$$

then a positive solution $y(t)$ of $(E)$ does not satisfy $\left(N_{1}\right)-\left(N_{4}\right)$ of Lemma 3.

Proof Assume on the contrary that $y(t)$ is an eventually positive solution of $(E)$ satisfying condition $\left(N_{1}\right)$ or $\left(N_{4}\right)$ of Lemma 3 for $t \geq t_{1} \geq t_{0}$. Since $y(t)$ is positive and nondecreasing, there exists a positive constant $k>0$ such that $y(t) \geq k$ for $t \geq t_{1}$.

Integrating $(E)$ from $t_{1}$ to $\infty$, we get

$$
L_{3} y\left(t_{1}\right) \geq \int_{t_{1}}^{\infty} p(s) y(\tau(s)) \mathrm{d} s \geq k \int_{t_{1}}^{\infty} p(s) \mathrm{d} s,
$$

which is a contradiction with respect to (2.1).

Now, we assume that $y(t)$ is an eventually positive solution of $(E)$ satisfying condition $\left(N_{2}\right)$ of Lemma 3 for $t \geq t_{1}$. Integrating $(E)$ from $t_{1}$ to $t$ and using that $y(t)$ is positive and nondecreasing, we get

$$
-L_{3} y(t) \geq k \int_{t_{1}}^{t} p(s) \mathrm{d} s
$$

Integrating the above inequality from $t_{1}$ to $\infty$, we obtain

$$
L_{2} y\left(t_{1}\right) \geq k \int_{t_{1}}^{\infty} \frac{1}{r_{3}(u)} \int_{t_{1}}^{u} p(s) \mathrm{d} s \mathrm{~d} u=k \int_{t_{1}}^{\infty} \pi_{3}(u) p(u) \mathrm{d} u,
$$

which contradicts (2.1). 
Finally, we assume that $y(t)$ is an eventually positive solution of $(E)$ satisfying condition $\left(N_{3}\right)$ of Lemma 3 for $t \geq t_{1}$. Similarly as above, we are led to (2.2). Integrating this from $t_{1}$ to $t$, we obtain

$$
-L_{2} y(t) \geq k \int_{t_{1}}^{t} \frac{1}{r_{3}(u)} \int_{t_{1}}^{u} p(s) \mathrm{d} s \mathrm{~d} u
$$

An integration from $t_{1}$ to $\infty$ yields

$$
\begin{aligned}
L_{1} y\left(t_{1}\right) & \geq k \int_{t_{1}}^{\infty} \frac{1}{r_{2}(v)} \int_{t_{1}}^{v} \frac{1}{r_{3}(u)} \int_{t_{1}}^{u} p(s) \mathrm{d} s \mathrm{~d} u \mathrm{~d} v \\
& =k \int_{t_{1}}^{\infty} \frac{1}{r_{3}(u)} \int_{t_{1}}^{u} p(s) \mathrm{d} s \int_{u}^{\infty} \frac{1}{r_{2}(v)} \mathrm{d} v \mathrm{~d} u \\
& =k \int_{t_{1}}^{\infty} \frac{1}{r_{3}(u)} \pi_{2}(u) \int_{t_{1}}^{u} p(s) \mathrm{d} s \mathrm{~d} u=k \int_{t_{1}}^{\infty} \pi_{32}(s) p(s) \mathrm{d} s,
\end{aligned}
$$

which is a contradiction and the proof is finished.

Theorem 2 If

$$
\int_{t_{1}}^{\infty} \pi_{12}(\tau(s)) p(s) \mathrm{d} s=\infty
$$

then the positive solution $y(t)$ of $(E)$ does not satisfy $\left(N_{5}\right),\left(N_{6}\right)$ of Lemma 3.

Proof Assume on the contrary that $y(t)$ is an eventually positive solution of $(E)$ satisfying condition $\left(N_{5}\right)$ of Lemma 3 for $t \geq t_{1} \geq t_{0}$. Since $L_{2} y(t)$ is a positive and increasing function there exists a positive constant $k>0$ such that

$$
L_{2} y(t) \geq k
$$

for $t \geq t_{1}$. Integrating the previous inequality from $t$ to $\infty$, we have

$$
-r_{1}(t) y^{\prime}(t) \geq k \int_{t}^{\infty} \frac{1}{r_{2}(s)} \mathrm{d} s
$$

After integration from $\tau(t)$ to $\infty$, we get

$$
y(\tau(t)) \geq k \int_{\tau(t)}^{\infty} \frac{1}{r_{1}(u)} \int_{u}^{\infty} \frac{1}{r_{2}(s)} \mathrm{d} s \mathrm{~d} u=k \pi_{12}(\tau(t)) .
$$

On the other hand, in view of (2.4), an integration of $(E)$ from $t_{1}$ to $\infty$ yields

$$
L_{3} y\left(t_{1}\right) \geq \int_{t_{1}}^{\infty} p(s) y(\tau(s)) \mathrm{d} s \geq k \int_{t_{1}}^{\infty} p(s) \pi_{12}(\tau(s)) \mathrm{d} s
$$

which contradicts (2.3).

Now, we assume that $y(t)$ is an eventually positive solution of $(E)$ satisfying condition $\left(N_{6}\right)$ of Lemma 3 for $t \geq t_{1} \geq t_{0}$. Seeing that $L_{1}(y)$ is a negative and decreasing function, 
there exists a constant $k>0$ such that

$$
L_{1} y(t)=r_{1}(t) y^{\prime}(t) \leq-k
$$

for $t \geq t_{1}$, and integrating this inequality from $\tau(t)$ to $\infty$, we have

$$
y(\tau(t)) \geq k \int_{\tau(t)}^{\infty} \frac{1}{r_{1}(s)} \mathrm{d} s .
$$

Integrating $(E)$ from $t_{1}$ to $\infty$ and using (2.5), we obtain

$$
\begin{aligned}
L_{3} y\left(t_{1}\right) & \geq \int_{t_{1}}^{\infty} p(s) y(\tau(s)) \mathrm{d} s \geq k \int_{t_{1}}^{\infty} p(s) \int_{\tau(s)}^{\infty} \frac{1}{r_{1}(u)} \mathrm{d} u \mathrm{~d} s \\
& =k \int_{t_{1}}^{\infty} p(s) \pi_{1}(\tau(s)) \mathrm{d} s,
\end{aligned}
$$

which is a contradiction to (2.3). The proof is completed.

Theorems 2.1 and 2.3 reduce the number of possible nonoscillatory solutions of $(E)$ only to $\left(N_{A}\right)$ or $\left(N_{B}\right)$, which essentially simplifies examination of $(E)$.

\section{Main results}

Now we provide useful monotonic properties of nonoscillatory solutions of $(E)$ satisfying conditions $\left(N_{A}\right)$ or $\left(N_{B}\right)$ of Lemma 3 . We begin with the following auxiliary result.

Lemma 4 Assume that $y(t)$ is an eventually positive solution of $(E)$ satisfying condition $\left(N_{A}\right)$ of Lemma 3 and

$$
\int_{t_{0}}^{\infty} p(s) \pi_{3}(s) \pi_{1}(\tau(s)) \mathrm{d} s=\infty .
$$

Then

$$
\lim _{t \rightarrow \infty} r_{1}(t) y^{\prime}(t)=\lim _{t \rightarrow \infty} y(t)=0 .
$$

Proof Assume that $y(t)$ is an eventually positive solution of $(E)$ satisfying condition $\left(N_{A}\right)$ of Lemma 3 for $t \geq t_{1} \geq t_{0}$.

Since $y(t)$ is positive and decreasing, there exists $\lim _{t \rightarrow \infty} y(t)=\ell \geq 0$. We claim that $\ell=0$. If not, then $y(\tau(t)) \geq \ell>0$, eventually, let us say for $t \geq t_{1}$. An integration of $(E)$ from $t_{1}$ to $t$ yields

$$
-\left(L_{2} y(t)\right)^{\prime} \geq \frac{1}{r_{3}(t)} \int_{t_{1}}^{t} p(s) y(\tau(s)) \mathrm{d} s \geq \frac{\ell}{r_{3}(t)} \int_{t_{1}}^{t} p(s) \mathrm{d} s .
$$

Integrating from $t_{1}$ to $\infty$, we obtain

$$
\left(r_{2}\left(r_{1} y^{\prime}\right)\right)^{\prime}\left(t_{1}\right) \geq \ell \int_{t_{1}}^{\infty} \frac{1}{r_{3}(u)} \int_{t_{1}}^{u} p(s) \mathrm{d} s \mathrm{~d} u=\ell \int_{t_{1}}^{\infty} p(s) \pi_{3}(s) \mathrm{d} s,
$$

which contradicts (3.1), and we conclude that $y(t) \rightarrow 0$ as $t \rightarrow \infty$. 
On the other hand, since $-r_{1} y^{\prime}$ is positive and decreasing, there exists

$$
\lim _{t \rightarrow \infty}-r_{1} y^{\prime}(t)=\ell \geq 0
$$

We assume on the contrary that $\ell>0$. Then

$$
-r_{1} y^{\prime}(t)>\ell, \quad t \geq t_{1}
$$

Integrating from $t$ to $\infty$, one gets

$$
y(t) \geq \ell \pi_{1}(t)
$$

which setting into (3.3) yields

$$
-\left(L_{2} y(t)\right)^{\prime} \geq \frac{\ell}{r_{3}(t)} \int_{t_{1}}^{t} p(s) \pi_{1}(\tau(s)) \mathrm{d} s
$$

An integration from $t_{1}$ to $\infty$ yields

$$
\left(r_{2}\left(r_{1} y^{\prime}\right)\right)^{\prime}\left(t_{1}\right) \geq \ell \int_{t_{1}}^{\infty} \frac{1}{r_{3}(u)} \int_{t_{1}}^{u} p(s) \pi_{1}(\tau(s)) \mathrm{d} s \mathrm{~d} u=\ell \int_{t_{1}}^{\infty} p(s) \pi_{3}(s) \pi_{1}(\tau(s)) \mathrm{d} s .
$$

This is a contradiction, and the proof is complete now.

Theorem 3 Let (3.1) hold. Assume that $y(t)$ is an eventually positive solution of $(E)$ satisfying condition $\left(N_{A}\right)$ of Lemma 3. Then

$$
\begin{aligned}
& \frac{y(t)}{\pi_{12}(t)} \text { is decreasing, } \\
& \frac{y(t)}{\pi_{123}(t)} \text { is increasing. }
\end{aligned}
$$

Proof Assume that $y(t)$ is an eventually positive solution of $(E)$ satisfying condition $\left(N_{A}\right)$ of Lemma 3 for $t \geq t_{1} \geq t_{0}$. At first, we shall show that $\frac{y(t)}{\pi_{12}(t)}$ is decreasing. Employing (3.2) and using that $L_{2} y(t)$ is positive and decreasing, we have

$$
-r_{1}(t) y^{\prime}(t)=\int_{t}^{\infty} \frac{L_{2} y(s)}{r_{2}(s)} \mathrm{d} s \leq L_{2} y(t) \pi_{2}(t)
$$

which implies

$$
\left(\frac{r_{1}(t) y^{\prime}(t)}{\pi_{2}(t)}\right)^{\prime}=\frac{L_{2} y(t) \pi_{2}(t)+r_{1}(t) y^{\prime}(t)}{r_{2}(t) \pi_{2}^{2}(t)} \geq 0
$$

Thus, $\frac{r_{1}(t) y^{\prime}(t)}{\pi \pi_{2}(t)}$ is increasing, and in view of (3.2), we get

$$
-y(t)=\int_{t}^{\infty} \frac{r_{1}(s) y^{\prime}(s)}{\pi_{2}(s)} \frac{\pi_{2}(s)}{r_{1}(s)} \mathrm{d} s \geq \frac{r_{1}(t) y^{\prime}(t)}{\pi_{2}(t)} \pi_{12}(t)
$$


which yields

$$
\left(\frac{y(t)}{\pi_{12}(t)}\right)^{\prime}=\frac{r_{1}(t) y^{\prime}(t) \pi_{12}(t)+y(t) \pi_{2}(t)}{r_{1}(t) \pi_{12}^{2}(t)} \leq 0,
$$

and we conclude that $\frac{y(t)}{\pi} \pi_{12}(t)$ is a decreasing function.

Now, we shall prove that $\frac{y(t)}{\pi_{123}(t)}$ is an increasing function. Employing that $L_{3} y(t)$ is a negative and decreasing function, we have

$$
-L_{2} y(t) \leq \int_{t}^{\infty} \frac{L_{3} y(s)}{r_{3}(s)} \mathrm{d} s \leq L_{3} y(t) \pi_{3}(t)
$$

which yields

$$
\left(\frac{L_{2} y(t)}{\pi_{3}(t)}\right)^{\prime}=\frac{L_{3} y(t) \pi_{3}(t)+L_{2} y(t)}{r_{3}(t) \pi_{3}^{2}(t)} \geq 0
$$

and $\frac{L_{2} y(t)}{\pi_{3}(t)}$ is increasing. Therefore,

$$
-r_{1}(t) y^{\prime}(t)=\int_{t}^{\infty} \frac{L_{2} y(s)}{\pi_{3}(s)} \frac{\pi_{3}(s)}{r_{2}(s)} \mathrm{d} s \geq \frac{L_{2} y(t)}{\pi_{3}(t)} \pi_{23}(t) .
$$

This inequality implies that $\frac{r_{1}(t) y^{\prime}(t)}{\pi_{23}(t)}$ is increasing. Finally,

$$
-y(t)=\int_{t}^{\infty} \frac{r_{1}(s) y^{\prime}(s)}{\pi_{23}(s)} \frac{\pi_{23}(s)}{r_{1}(s)} \mathrm{d} s \leq \frac{r_{1}(t) y^{\prime}(t) \pi_{123}(t)}{\pi_{23}(t)},
$$

which implies

$$
\left(\frac{y(t)}{\pi_{123}(t)}\right)^{\prime}=\frac{r_{1}(t) y^{\prime}(t) \pi_{123}(t)+y(t) \pi_{23}(t)}{r_{1}(t) \pi_{123}^{2}(t)} \geq 0,
$$

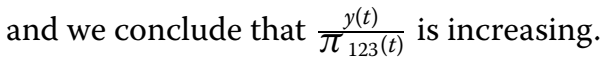

Theorem 4 Assume that $y(t)$ is an eventually positive solution of $(E)$ satisfying condition $\left(N_{B}\right)$ of Lemma 3. Then

$$
\frac{y(t)}{\pi_{1}(t)} \quad \text { is increasing. }
$$

Proof Assume that $y(t)$ is an eventually positive solution of $(E)$ satisfying condition $\left(N_{B}\right)$ of Lemma 3 for $t \geq t_{1} \geq t_{0}$. Applying the monotonic property of $r_{1}(t) y^{\prime}(t)$, we get

$$
-y(t)=\int_{t}^{\infty} \frac{r_{1}(s) y^{\prime}(s)}{r_{1}(s)} \mathrm{d} s \leq r_{1}(t) y^{\prime}(t) \pi_{1}(t),
$$

which gives

$$
\left(\frac{y(t)}{\pi_{1}(t)}\right)^{\prime}=\frac{r_{1}(t) y^{\prime}(t) \pi_{1}(t)+y(t)}{r_{1}(t) \pi_{1}^{2}(t)} \geq 0,
$$

and we conclude that $\frac{y(t)}{\pi_{1}(t)}$ is increasing. 
Now, we are prepared for establishing the criteria for the essential classes $\left(N_{A}\right)$ and $\left(N_{B}\right)$ to be empty.

Theorem 5 Let (3.1) hold. If

$$
\begin{aligned}
& \limsup _{t \rightarrow \infty}\left\{\frac{\pi_{123}(\tau(t))}{\pi_{12}(\tau(t))} \int_{t_{1}}^{\tau(t)} p(s) \pi_{12}(\tau(s)) \mathrm{d} s+\frac{1}{\pi_{12}(\tau(t))} \int_{\tau(t)}^{t} p(s) G(s, \tau(t)) \pi_{12}(\tau(s)) \mathrm{d} s\right. \\
& \left.+\frac{1}{\pi_{123}(\tau(t))} \int_{t}^{\infty} p(s) G(s, \tau(t)) \pi_{123}(\tau(s)) \mathrm{d} s\right\}>1
\end{aligned}
$$

where

$$
G(s, t)=\pi_{321}(s)+\pi_{12}(t) \pi_{3}(s)-\pi_{1}(t) \pi_{32}(s),
$$

then the class $\left(N_{A}\right)$ of Lemma 3 is empty.

Proof Assume on the contrary that $y(t)$ is an eventually positive solution of $(E)$ satisfying condition $\left(N_{A}\right)$ of Lemma 3 for $t \geq t_{1} \geq t_{0}$. Integrating $(E)$ twice from $t_{1}$ to $t$ and from $t$ to $\infty$, we obtain

$$
L_{2} y(t) \geq \int_{t}^{\infty} \frac{1}{r_{3}(u)} \int_{t_{1}}^{u} p(s) y(\tau(s)) \mathrm{d} s \mathrm{~d} u .
$$

Changing the order of integrating in the previous inequality, we see

$$
\left(r_{1}(t) y^{\prime}(t)\right)^{\prime} \geq \frac{\pi_{3}(t)}{r_{2}(t)} \int_{t_{1}}^{t} p(s) y(\tau(s)) \mathrm{d} s+\frac{1}{r_{2}(t)} \int_{t}^{\infty} p(s) y(\tau(s)) \pi_{3}(s) \mathrm{d} s .
$$

Integrating the above inequality from $t$ to $\infty$, one gets

$$
\begin{aligned}
-r_{1}(t) y^{\prime}(t) \geq & \int_{t}^{\infty} \frac{\pi_{3}(u)}{r_{2}(u)} \int_{t_{1}}^{u} p(s) y(\tau(s)) \mathrm{d} s \mathrm{~d} u+\int_{t}^{\infty} \frac{1}{r_{2}(u)} \int_{u}^{\infty} p(s) y(\tau(s)) \pi_{3}(s) \mathrm{d} s \mathrm{~d} u \\
= & \pi_{23}(t) \int_{t_{1}}^{t} p(s) y(\tau(s)) \mathrm{d} s+\int_{t}^{\infty} \pi_{23}(s) p(s) y(\tau(s)) \mathrm{d} s \\
& +\int_{t}^{\infty} p(s) y(\tau(s)) \pi_{3}(s)\left[\pi_{2}(t)-\pi_{2}(s)\right] \mathrm{d} s .
\end{aligned}
$$

It follows from Lemma 1 that $\pi_{23}(s)+\pi_{32}(s)=\pi_{2}(s) \pi_{3}(s)$, and so

$$
\begin{aligned}
-y^{\prime}(t)= & \frac{\pi_{23}(t)}{r_{1}(t)} \int_{t_{1}}^{t} p(s) y(\tau(s)) \mathrm{d} s+\frac{\pi_{2}(t)}{r_{1}(t)} \int_{t}^{\infty} p(s) y(\tau(s)) \pi_{3}(s) \mathrm{d} s \\
& -\frac{1}{r_{1}(t)} \int_{t}^{\infty} p(s) y(\tau(s)) \pi_{32}(s) \mathrm{d} s .
\end{aligned}
$$

Integrating once more from $t$ to $\infty$ and employing Lemma 2 , we have

$$
\begin{aligned}
y(t) \geq & \pi_{123}(t) \int_{t_{1}}^{t} p(s) y(\tau(s)) \mathrm{d} s+\int_{t}^{\infty} p(s) y(\tau(s)) \pi_{123}(s) \mathrm{d} s \\
& +\int_{t}^{\infty} p(s) y(\tau(s)) \pi_{3}(s)\left[\pi_{12}(t)-\pi_{12}(s)\right] \mathrm{d} s
\end{aligned}
$$




$$
\begin{aligned}
& -\int_{t}^{\infty} p(s) y(\tau(s)) \pi_{32}(s)\left[\pi_{1}(t)-\pi_{1}(s)\right] \mathrm{d} s \\
= & \pi_{123}(t) \int_{t_{1}}^{t} p(s) y(\tau(s)) \mathrm{d} s+\int_{t}^{\infty} p(s) y(\tau(s)) \pi_{321}(s) \mathrm{d} s \\
& +\pi_{12}(t) \int_{t}^{\infty} p(s) y(\tau(s)) \pi_{3}(s) \mathrm{d} s-\pi_{1}(t) \int_{t}^{\infty} p(s) y(\tau(s)) \pi_{32}(s) \mathrm{d} s \\
= & \pi_{123}(t) \int_{t_{1}}^{t} p(s) y(\tau(s)) \mathrm{d} s+\int_{t}^{\infty} p(s) y(\tau(s)) G(s, t) \mathrm{d} s .
\end{aligned}
$$

Then

$$
\begin{aligned}
y(\tau(t)) \geq & \pi_{123}(\tau(t)) \int_{t_{1}}^{\tau(t)} p(s) y(\tau(s)) \mathrm{d} s+\int_{\tau(t)}^{\infty} p(s) y(\tau(s)) G(s, \tau(t)) \mathrm{d} s \\
= & \pi_{123}(\tau(t)) \int_{t_{1}}^{\tau(t)} p(s) y(\tau(s)) \mathrm{d} s+\int_{\tau(t)}^{t} p(s) y(\tau(s)) G(s, \tau(t)) \mathrm{d} s \\
& +\int_{t}^{\infty} p(s) y(\tau(s)) G(s, \tau(t)) \mathrm{d} s .
\end{aligned}
$$

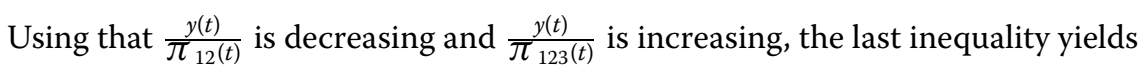

$$
\begin{aligned}
y(\tau(t)) \geq & \pi_{123}(\tau(t)) \frac{y(\tau(t))}{\pi_{12}(\tau(t))} \int_{t_{1}}^{\tau(t)} p(s) \pi_{12}(\tau(s)) \mathrm{d} s \\
& +\frac{y(\tau(t))}{\pi_{12}(\tau(t))} \int_{\tau(t)}^{t} p(s) G(s, \tau(t)) \pi_{12}(\tau(s)) \mathrm{d} s \\
& +\frac{y(\tau(t))}{\pi_{123}(\tau(t))} \int_{t}^{\infty} p(s) G(s, \tau(t)) \pi_{123}(\tau(s)) \mathrm{d} s .
\end{aligned}
$$

Then

$$
\begin{aligned}
1 \geq & \left\{\frac{\pi_{123}(\tau(t))}{\pi_{12}(\tau(t))} \int_{t_{1}}^{\tau(t)} p(s) \pi_{12}(\tau(s)) \mathrm{d} s+\frac{1}{\pi_{12}(\tau(t))} \int_{\tau(t)}^{t} p(s) G(s, \tau(t)) \pi_{12}(\tau(s)) \mathrm{d} s\right. \\
& \left.+\frac{1}{\pi_{123}(\tau(t))} \int_{t}^{\infty} p(s) G(s, \tau(t)) \pi_{123}(\tau(s)) \mathrm{d} s\right\}
\end{aligned}
$$

which contradicts (3.7).

\section{Theorem 6 If}

$$
\begin{gathered}
\limsup _{t \rightarrow \infty}\left\{\int_{t_{1}}^{\tau(t)} p(s) H(s, \tau(t)) \mathrm{d} s+\int_{\tau(t)}^{t} p(s) \pi_{321}(s) \mathrm{d} s\right. \\
\left.\quad+\frac{1}{\pi_{1}(\tau(t))} \int_{t}^{\infty} p(s) \pi_{321}(s) \pi_{1}(\tau(s)) \mathrm{d} s\right\}>1,
\end{gathered}
$$

where

$$
H(s, t)=\pi_{32}(s) \pi_{1}(t)+\pi_{3}(s) \pi_{12}(t)+\pi_{123}(t),
$$

then the class $\left(N_{B}\right)$ of Lemma 3 is empty. 
Proof Assume on the contrary that $y(t)$ is an eventually positive solution of $(E)$ satisfying condition $\left(N_{B}\right)$ of Lemma 3 for $t \geq t_{1} \geq t_{0}$. Integrating $(E)$ twice from $t_{1}$ to $t$ and thereafter switching the order of integration, we obtain

$$
-\left(r_{1}(t) y^{\prime}(t)\right)^{\prime} \geq \frac{1}{r_{2}(t)} \int_{t_{1}}^{t} p(s) y(\tau(s))\left(\pi_{3}(s)-\pi_{3}(t)\right) \mathrm{d} s .
$$

Integrating the above inequality again from $t_{1}$ to $t$ and changing the order of integration, we get

$$
\begin{aligned}
-r_{1}(t) y^{\prime}(t) \geq & \int_{t_{1}}^{t} \frac{1}{r_{2}(u)} \int_{t_{1}}^{u} p(s) y(\tau(s))\left(\pi_{3}(s)-\pi_{3}(t)\right) \mathrm{d} s \mathrm{~d} u \\
= & \int_{t_{1}}^{t} p(s) y(\tau(s)) \pi_{3}(s)\left[\pi_{2}(s)-\pi_{2}(t)\right] \mathrm{d} s \\
& -\int_{t_{1}}^{t} p(s) y(\tau(s))\left[\pi_{23}(s)-\pi_{23}(t)\right] \mathrm{d} s .
\end{aligned}
$$

Applying Lemma 1, we can write

$$
-r_{1}(t) y^{\prime}(t) \geq \int_{t_{1}}^{t} p(s) y(\tau(s))\left[\pi_{32}(s)-\pi_{3}(s) \pi_{2}(t)+\pi_{23}(t)\right] \mathrm{d} s .
$$

Integrating the previous inequality from $t$ to $\infty$ and consequently switching the order of integration, we obtain

$$
\begin{aligned}
y(t) \geq & \int_{t}^{\infty} \frac{1}{r_{1}(u)} \int_{t_{1}}^{u} p(s) y(\tau(s))\left[\pi_{32}(s)-\pi_{3}(s) \pi_{2}(u)+\pi_{23}(u)\right] \mathrm{d} s \mathrm{~d} u \\
= & \int_{t_{1}}^{t} p(s) y(\tau(s)) \pi_{32}(s) \pi_{1}(t) \mathrm{d} s-\int_{t_{1}}^{t} p(s) y(\tau(s)) \pi_{3}(s) \pi_{12}(t) \mathrm{d} s \\
& +\int_{t_{1}}^{t} p(s) y(\tau(s)) \pi_{123}(t) \mathrm{d} s+\int_{t}^{\infty} p(s) y(\tau(s)) \pi_{32}(s) \pi_{1}(s) \mathrm{d} s \\
& -\int_{t}^{\infty} p(s) y(\tau(s)) \pi_{3}(s) \pi_{12}(s) \mathrm{d} s+\int_{t}^{\infty} p(s) y(\tau(s)) \pi_{123}(s) \mathrm{d} s .
\end{aligned}
$$

Employing the equality $\pi_{123}(t)+\pi_{32}(t) \pi_{1}(t)-\pi_{3}(t) \pi_{12}(t)=\pi_{321}(t)$, we can rewrite the above inequality into a simpler form

$$
\begin{aligned}
y(t) \geq & \int_{t_{1}}^{t} p(s) y(\tau(s))\left[\pi_{32}(s) \pi_{1}(t)-\pi_{3}(s) \pi_{12}(t)+\pi_{123}(t)\right] \mathrm{d} s \\
& +\int_{t}^{\infty} p(s) y(\tau(s)) \pi_{321}(s) \mathrm{d} s .
\end{aligned}
$$

Using notation for $H(s, t)$

$$
y(t) \geq \int_{t_{1}}^{t} p(s) y(\tau(s)) H(s, t) \mathrm{d} s+\int_{t}^{\infty} p(s) y(\tau(s)) \pi_{321}(s) \mathrm{d} s,
$$


then

$$
\begin{aligned}
y(\tau(t)) \geq & \int_{t_{1}}^{\tau(t)} p(s) y(\tau(s)) H(s, \tau(t)) \mathrm{d} s+\int_{\tau(t)}^{\infty} p(s) y(\tau(s)) \pi_{321}(s) \mathrm{d} s \\
= & \int_{t_{1}}^{\tau(t)} p(s) y(\tau(s)) H(s, \tau(t)) \mathrm{d} s+\int_{\tau(t)}^{t} p(s) y(\tau(s)) \pi_{321}(s) \mathrm{d} s \\
& +\int_{t}^{\infty} p(s) y(\tau(s)) \pi_{321}(s) \mathrm{d} s .
\end{aligned}
$$

Applying the monotonic properties of $y(t)$ (decreasing) and $\frac{y(t)}{\pi_{1}(t)}$ (increasing), we have

$$
\begin{aligned}
y(\tau(t)) \geq & y(\tau(t)) \int_{t_{1}}^{\tau(t)} p(s) H(s, \tau(t)) \mathrm{d} s+y(\tau(t)) \int_{\tau(t)}^{t} p(s) \pi_{321}(s) \mathrm{d} s \\
& +\frac{y(\tau(t))}{\pi_{1}(\tau(t))} \int_{t}^{\infty} p(s) \pi_{1}(\tau(s)) \pi_{321}(s) \mathrm{d} s,
\end{aligned}
$$

which implies

$$
\begin{aligned}
1 \geq & \int_{t_{1}}^{\tau(t)} p(s) H(s, \tau(t)) \mathrm{d} s+\int_{\tau(t)}^{t} p(s) \pi_{321}(s) \mathrm{d} s \\
& +\frac{1}{\pi_{1}(\tau(t))} \int_{t}^{\infty} p(s) \pi_{1}(\tau(s)) \pi_{321}(s) \mathrm{d} s .
\end{aligned}
$$

This contradicts (3.8), and the proof is complete.

The following result is intended to avoid evaluation of function $H(s, t)$ and to simplify criterion (3.7).

\section{Corollary 1 If}

$$
\begin{gathered}
\limsup _{t \rightarrow \infty}\left\{\pi_{321}(\tau(t)) \int_{t_{1}}^{\tau(t)} p(s) \mathrm{d} s+\int_{\tau(t)}^{t} p(s) \pi_{321}(s) \mathrm{d} s\right. \\
\left.+\frac{1}{\pi_{1}(\tau(t))} \int_{t}^{\infty} p(s) \pi_{321}(s) \pi_{1}(\tau(s)) \mathrm{d} s\right\}>1,
\end{gathered}
$$

then the class $\left(N_{B}\right)$ of Lemma 3 is empty.

Proof Assume on the contrary that $y(t)$ is an eventually positive solution of $(E)$ satisfying condition $\left(N_{B}\right)$ of Lemma 3 for $t \geq t_{1} \geq t_{0}$. Proceeding similarly as in the proof of Theorem 6, we get (3.9). It follows from monotonic properties of $H(s, t)$ and Lemma 2 that

$$
H(s, t) \geq H(t, t)=\pi_{321}(t) \quad \text { for } s \in\left\langle t_{1}, t\right\rangle .
$$

Using (3.11) in (3.9), we obtain

$$
y(t) \geq \pi_{321}(t) \int_{t_{1}}^{t} p(s) y(\tau(s)) \mathrm{d} s+\int_{t}^{\infty} p(s) y(\tau(s)) \pi_{321}(s) \mathrm{d} s .
$$


Then

$$
\begin{aligned}
y(\tau(t)) \geq & \pi_{321}(\tau(t)) \int_{t_{1}}^{\tau(t)} p(s) y(\tau(s)) \mathrm{d} s+\int_{\tau(t)}^{t} p(s) y(\tau(s)) \pi_{321}(s) \mathrm{d} s \\
& +\int_{t}^{\infty} p(s) y(\tau(s)) \pi_{321}(s) \mathrm{d} s .
\end{aligned}
$$

Taking into account that $y(t)$ is decreasing and $\frac{y(t)}{\pi_{1}(t)}$ is increasing finally, we have

$$
\begin{aligned}
1 \geq & \pi_{321}(\tau(t)) \int_{t_{1}}^{\tau(t)} p(s) \mathrm{d} s+\int_{\tau(t)}^{t} p(s) \pi_{321}(s) \mathrm{d} s \\
& +\frac{1}{\pi_{1}(\tau(t))} \int_{t}^{\infty} p(s) \pi_{321}(s) \pi_{1}(\tau(s)) \mathrm{d} s,
\end{aligned}
$$

which contradicts the assumption of the corollary.

Picking up the previous results, we can establish easily verifiable oscillatory criteria.

Theorem 7 Let (2.1), (3.1), (3.7), (3.8) hold. Then (E) is oscillatory.

Theorem 8 Let (2.1), (3.1), (3.7), (3.10) hold. Then (E) is oscillatory.

We support our results with an illustrative example, in which also some comparison with existing latest ones is made.

Example 1 Let us consider noncanonical fourth-order delay differential equation in the form

$$
\left(\left(t^{2}\left(t^{2}\left(t^{2} y^{\prime}(t)\right)^{\prime}\right)^{\prime}\right)^{\prime}+a t^{2} y(\lambda t)=0, \quad t \geq t_{0}>0\right.
$$

where $a>0, \lambda \in(0,1), \pi_{i}(t)=1 / t, \pi_{i j}(t)=1 /\left(2 t^{2}\right), \pi_{123}(t)=\pi_{321}(t)=1 /\left(6 t^{3}\right)$. It is easy to verify that (2.1), (3.1) hold.

Condition (3.7) takes the form

$$
\frac{a}{36 \lambda^{2}}\left(9+9 \lambda-\lambda^{2}+18 \ln \frac{1}{\lambda}\right)>1
$$

Condition (3.8) takes the form

$$
a\left(\frac{17}{6}+\ln \frac{1}{\lambda}\right)>6
$$

By Theorem 7, Eq. $\left(E_{x}\right)$ is oscillatory provided that both (3.12) and (3.13) hold. In particular case where $\lambda=0.8$, conditions (3.12) and (3.13) reduce to $a>1.963$. The oscillatory criterion obtained by a different technique presented in paper [4] gives oscillation of $\left(E_{x}\right)$ if $a>7.913$.

On the other hand, for $\lambda=0.9$, Theorem 7 guarantees oscillation of Eq. $\left(E_{x}\right)$ provided that $a>1.77$, while the best criterion from [7] requires $a>3.50$.

So our results are more efficient than the previous ones. 


\section{Acknowledgements}

The authors express their sincere gratitude to the editors for the careful reading of the original manuscript and useful comments that helped to improve the presentation of the results and accentuate important details.

Funding

The paper has been supported by the grant project KEGA 035TUKE-4/2017.

Availability of data and materials

Data sharing not applicable to this article as no datasets were generated or analysed during the current study.

\section{Competing interests}

The authors declare that they have no competing interests.

\section{Authors' contributions}

Both authors contributed equally to this work. They read and approved the final version of the manuscript.

\section{Publisher's Note}

Springer Nature remains neutral with regard to jurisdictional claims in published maps and institutional affiliations.

Received: 18 June 2019 Accepted: 29 August 2019 Published online: 11 September 2019

\section{References}

1. Baculikova, B., Dzurina, J., Jadlovska, I.: On asymptotic properties of solutions to third-order delay differential equations. Electron. J. Qual. Theory Differ. Equ. 2019, 7 (2019)

2. Bartusek, M., Dosla, Z.: Asymptotic problems for fourth-order nonlinear differential equations. Bound. Value Probl. 2013, 89 (2013)

3. Dzurina, J.: Comparison theorems for nonlinear ODE's. Math. Slovaca 42, 299-315 (1992)

4. Dzurina, J., Baculikova, B.: The fourth order strongly noncanonical operators. Open Math. 16, 1667-1674 (2018)

5. Dzurina, J., Jadlovska, I.: Oscillation of third-order differential equations with noncanonical operators. Appl. Math. Comput. 336, 394-402 (2018)

6. Dzurina, J., Kotorova, R.: Zero points of the solutions of a differential equation. Acta Electrotechn. Inform. 7, 26-29 (2007)

7. Grace, S.R., Dzurina, J., Jadlovska, I., Li, T.: On the oscillation of fourth-order delay differential equations. Adv. Differ. Equ. 2019, 118 (2019)

8. Jadlovska, I.: Application of Lambert W function in oscillation theory. Acta Electrotechn. Inform. 14(14), 9-17 (2014)

9. Kiguradze, I.T., Chanturia, T.A.: Asymptotic Properties of Solutions of Nonautonomous Ordinary Differential Equations. Kluwer Academic, Dordrecht (1993)

10. Kitamura, Y., Kusano, T.: Oscillations of first-order nonlinear differential equations with deviating arguments. Proc. Am. Math. Soc. 78, 64-68 (1980)

11. Koplatadze, R., Kvinkadze, G., Stavroulakis, I.P.: Properties $A$ and $B$ of $n$th order linear differential equations with deviating argument. Georgian Math. J. 6(6), 553-566 (1999)

12. Kusano, T., Naito, M.: Comparison theorems for functional differential equations with deviating arguments. J. Math. Soc. Jpn. 3, 509-533 (1981)

13. Ladde, G.S., Lakshmikantham, V., Zhang, B.G.: Oscillation Theory of Differential Equations with Deviating Arguments. Dekker, New York (1987)

14. Li, T., Rogovchenko, Y.V., Zhang, C.: Oscillation of fourth-order quasilinear differential equations. Math. Bohem. 140 405-418 (2015)

15. Li, T., Zhang, C., Thandapani, E.: Asymptotic behavior of fourth-order neutral dynamic equations with noncanonical operators. Taiwan. J. Math. 18, 1003-1019 (2014)

16. Mahfoud, W.E.: Comparison theorems for delay differential equations. Pac. J. Math. 83(83), 187-197 (1979)

17. Philos, C.G.: On the existence of nonoscillatory solutions tending to zero at $\infty$ for differential equations with positive delay. Arch. Math. 36, 168-178 (1981). Zbl. 0463.34050

18. Trench, W.: Canonical forms and principal systems for general disconjugate equations. Trans. Am. Math. Soc. 184, 319-327 (1974)

19. Zhang, C., Agarwal, R.P., Bohner, M., Li, T.: Oscillation of fourth-order delay dynamic equations. Sci. China Math. 58, $143-160(2015)$

20. Zhang, C., Li, T., Agarwal, R.P., Bohner, M.: Oscillation results for fourth-order nonlinear dynamic equations. Appl. Math. Lett. 25, 2058-2065 (2012) 